\title{
Design of Electric Linear Load Simulator based on LabVIEW+PXI
}

\author{
Weidong Pan $^{1}$, Yuanxun Fan ${ }^{1}$, Jianjie Lei ${ }^{1}$, Zhiwei $\mathrm{Xu}^{2}$ and Jian $\mathrm{Tao}^{2}$ \\ ${ }^{1}$ School of Mechanical Engineering, Nanjing University of Science and Technology, Nanjing 210094, China \\ ${ }^{2}$ Shanghai Aerospace Control Technology Institute, Shanghai 201109, China
}

\begin{abstract}
In order to realize the hardware-in-the-loop (HIL) experiments of a certain type of electric linear actuator in the laboratory, an electric linear load simulator (ELLS) based on LabVIEW+PXI platform was developed to simulate the external force of the actuator under actual working conditions. In structural design, the ball screw is used to convert the PMSM torque into linear force. In hardware design, the system is built using the upper-lower computer system architecture based on PXI bus. In software design, the program is written in LabVIEW, which mainly includes the data processing and display program in the upper computer and the data acquisition program, the loading motor control program and the actuator control program in the lower computer (PXI), in order to ensure the loading accuracy of ELLS, control methods such as force closed-loop PID controller, position feedforward compensation and input feedforward compensation are also introduced in the loading motor program. Finally, the ELLS was built and the signal tracking tests were carried out. The experimental results shows that the loading accuracy can reach $93.5 \%$ and $90.3 \%$ when ELLS tracks $1000 \mathrm{~N}-2 \mathrm{~Hz}$ and $1000 \mathrm{~N}-5 \mathrm{~Hz}$ respectively, which proved that the accuracy of ELLS satisfies the "Double Ten Index".
\end{abstract}

\section{Introduction}

The electric linear actuator is mainly used for the adjustment of the position of missile launcher, the adjustment of the opening of solenoid valve, and the precise control of spacecraft rendezvous and docking [1]. In order to realize the hardware-in-the-loop (HIL) experiments of actuators in the laboratory, it is necessary to design a test system that can simulate the external force of the electric linear actuators.

Electric Linear Load Simulator (ELLS) is a complex electromechanical system with linear force as the output object. It is an important equipment in lots of hardwarein-the-loop (HIL) experiments. In the loading test experiments of actuators, ELLS mainly provides dynamic/static linear force, which can test the control performance and output accuracy of actuators.

The measurement and control system based on LabVIEW and PXI platform is widely used in the field of measurement and control. Wang et al. [2] designed a displacement detection system for industrial controller based on PXI platform; Li et al. [3] also designed a four channel measurement and control system based on LabVIEW and PXI platform, which can achieve loading test on four-channel actuators at the same time; Wang et al. [4] developed an electric torque loading system based on virtual instrument technology, which can also realize the loading test of four-channel actuators.

In order to realize the hardware-in-the-loop (HIL) experiments of a certain type of electric linear actuator in the laboratory, an ELLS based on LabVIEW and PXI chassis was developed. The computer with LabVIEW is used as the upper computer, and the PXI chassis is used as the lower computer, which is mainly used for realtime data acquisition and processing. Control methods such as the position feedforward compensation, the input feedforward compensation and the force closed-loop PID control are adopted, thereby improved the loading precision and dynamic performance of ELLS.

\section{Hardware structure}

An ELLS is developed in our laboratory. It mainly consists of the upper computer (PC), the lower computer (PXI chassis), the loading platform and the sensor module. Its structure is shown in Fig. 1.

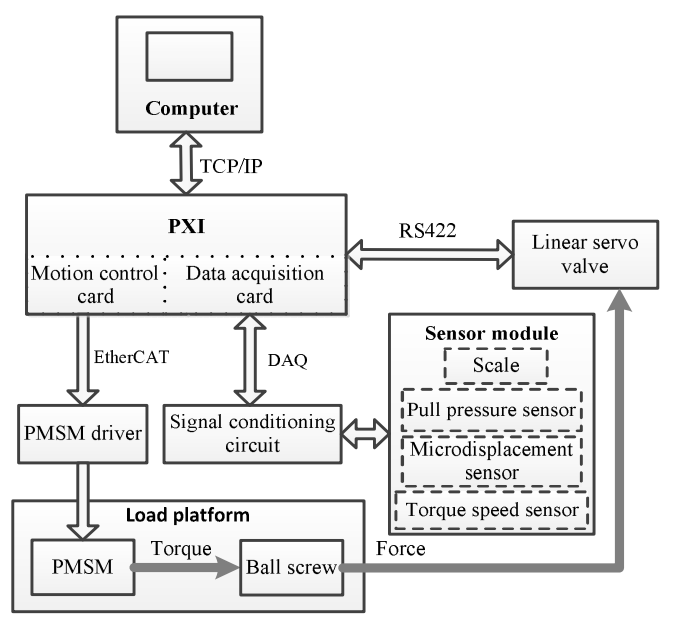

Fig. 1. Hardware Structure of ELLS. 


\subsection{Upper computer}

The PC is the core unit of the whole system. We use the high-speed industrial computer with LabVIEW software to control the process of the whole system. Its main functions include: accepting the data transmitted by the lower computer (PXI), generating and sending instructions, data processing and display etc.

\subsection{Lower computer}

The NI PXIe-8840RT real-time controller is selected as the lower computer of the system. The NI PXIe-8840RT real-time controller is installed in the NI PXIe-1071 chassis. In addition, the NI PXI-8231 Ethernet card and the NI PXIe-6341 data acquisition card are inserted into the 1071 chassis. The Ethernet card is used for the communication between the PXI and the loading motor. The data acquisition card has built-in A/D conversion circuit, which can realize multi-channel analog signal acquisition. The main functions of PXI are: loading motor control, motion control of the actuator, and realtime data acquisition and transmission.

\subsection{Platform for ELLS}

The loading module consists of permanent magnet synchronous motor (PMSM), torque speed sensor, coupling, ball screw and force sensor. The PMSM works in torque control mode, the output torque of PMSM passes through the torque speed sensor, the coupling and the ball screw respectively, and the torque is finally converted to linear force. At the same time, the force sensor detects the linear force in real time, the actuator and the ELLS are rigidly connected. The structure of the ELLS is shown in Figure 2.

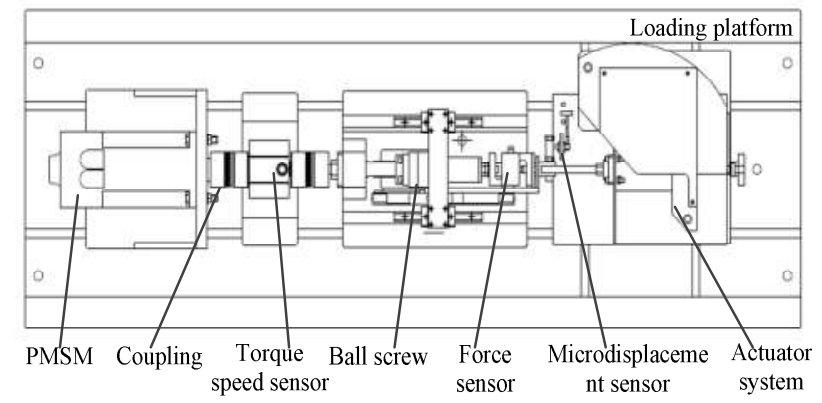

Fig. 2. Structure of the Platform for ELLS.

\subsection{Sensors}

For the test items of the actuator, we designed a set of sensor system, including linear grid ruler, force sensor, micro-displacement sensor and torque speed sensor. It is mainly used to test the transmission accuracy, mechanical rigidity and starting torque of the actuator.

\section{Software structure}

According to the design principle of LabVIEW software, the overall software architecture of the system is designed in the PC with LabVIEW software, the software structure is shown in Fig. 3.

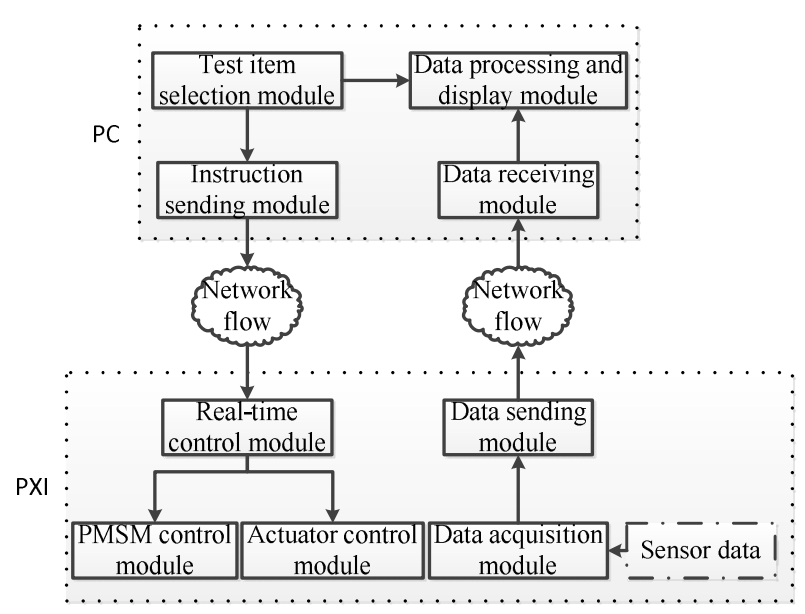

Fig. 3. Software structure of ELLS.

The application program is divided into two parts: the upper computer program and the lower computer program. The upper computer program is mainly used for the selection testing projects, receiving data from PXI, data processing and display, and sending control commands; The lower computer program is mainly responsible for sensor data acquisition, data transmission, and real-time control of PMSM; The network flow based on TCP/IP protocol is used for the data communication between PC and PXI.

\subsection{Upper computer program}

The upper computer program is divided into three parts: event handling loop, UI message loop, and data receiving loop. Each part of the loop can exchange messages and data through the message queue. The event handling loop is used for the selection of test items and the generation of control commands; UI message loop is used for data processing and display; the data receiving loop can receive the real-time data from the PXI and sent it to the UI message loop through the message queue. The program structure is shown in Fig. 4.

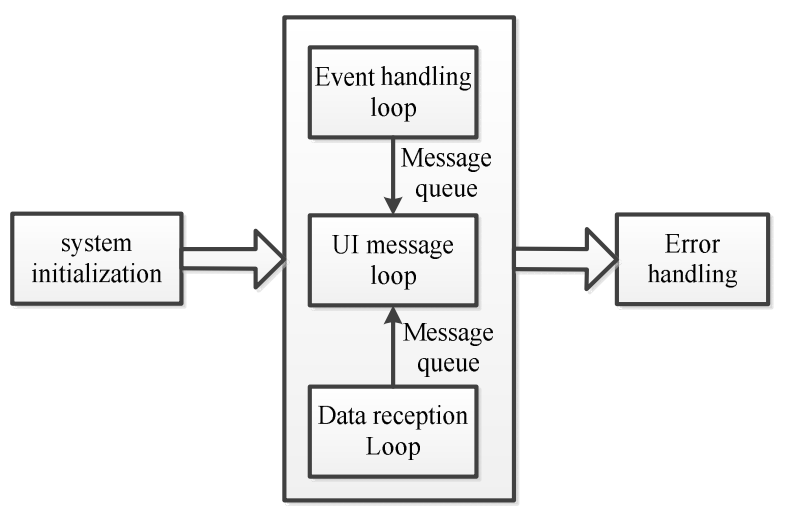

Fig. 4. Upper computer program structure.

\subsection{Lower computer program}


The lower computer program is mainly divided into data acquisition module and real-time control module. The data acquisition module is designed with DAQmax, which can realize Multi-channel single point sampling; The real-time control program is divided into actuator control module and PMSM control module.

\subsubsection{Data acquisition module}

The data acquisition module is designed based on NI DAQmax, which can realize real-time acquisition of multi-channel data at the same time, and transmit data to the upper computer program for data processing and display through the network stream.

\subsubsection{Actuator control module}

The PXIe-8840RT and the linear actuator are connected by RS422 serial port, The linear actuator control program is built with the VISA serial communication control, By continuously inputting the position command to the actuator, the position control of the linear actuator can be realized.

\subsubsection{PMSM control module}

The PMSM control program is the core part of the entire program, The PMSM is controlled by EtherCAT communication method. In order to improve the loading accuracy of ELLS and suppress the surplus torque caused by the motion of the actuator, the position feedforward compensation, the input feedforward compensation and the force closed loop PID control are introduced [5]. The control methods are described in Section 4. The PMSM control program is shown in Fig. 5.

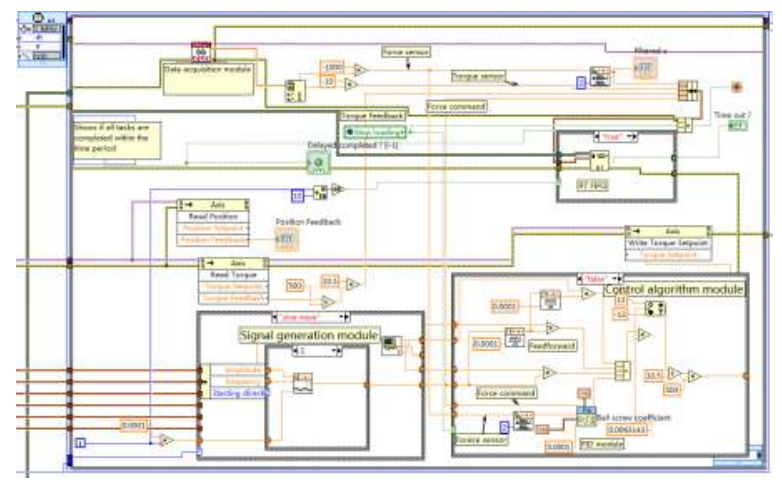

Fig. 5. PMSM control program.

\section{System control methods}

\subsection{System analysis}

The torque generated by PMSM is converted into the linear force through the ball screw pair to load the actuator, at the same time, the accuracy of the output force is affected by the actuator's motion, therefore, when modeling ELLS, the actuator's motion is generally equivalent to disturbance.

The ELLS model consists of two parts: the PMSM model and the ball screw model. The PMSM model is relatively mature, its vector control block diagram [6] is shown in Fig. 6.

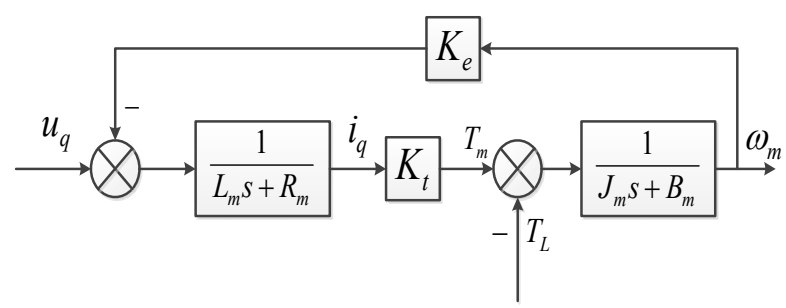

Fig. 6. PMSM control block diagram.

Where $u_{q}$ is the input voltage in $q$ axis; $K_{e}$ is back electromotive force constant; $J_{m}$ is the rotary inertia of the PMSM; $B_{m}$ is the damping coefficient; $T_{L}$ is the output torque of PMSM; $\omega_{m}$ is the angular velocity of the PMSM; $T_{m}$ is the electromagnetic torque of PMSM.

The second part is the ball screw. When modeling the ball screw, the output torque of PMSM is equal to the sum of the damping load, the inertial load and the elastic load. The equilibrium equation is as follows.

$$
T_{L}=J_{G} \frac{d^{2} \theta_{m}}{d t^{2}}+B_{G} \frac{d \theta_{m}}{d t}+K_{A}\left(\theta_{m}-\theta_{G}\right)
$$

$J_{G}$ is the moment of inertia of the ball screw; $B_{G}$ is the damping coefficient; $K_{A}$ is the elastic coefficient; $\theta_{m}$ is the output angle displacement of PMSM; $\theta_{G}$ is the angular displacement of the ball screw.

The conversion relationship between the input torque and the output torque of the ball screw is related to the helix angle $\gamma$, and the relationship between the input angular displacement and the output displacement is related to the lead (p) of the ball screw. Their expressions are [7].

$$
F=\frac{T_{L}}{R \tan \gamma}, \theta_{G}=\frac{2 \pi L}{P}
$$

The mathematical model of ELLS is shown in Fig. 7.

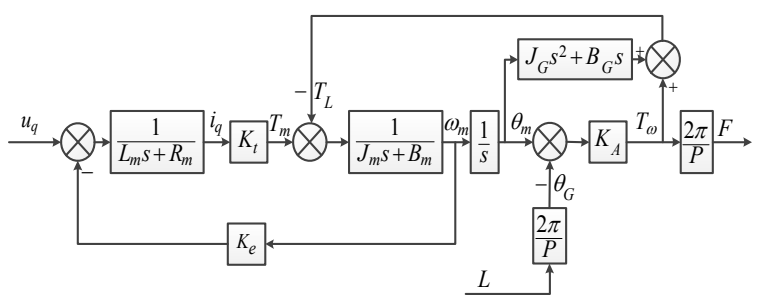

Fig. 7. The mathematical model of ELLS.

According to the ELLS control block diagram, the F is the output force which consists of two parts, One part is caused by the input voltage command, and the other part is the surplus force $Q$ caused by the position disturbance of the linear actuator [8], therefore, how to 
suppress the surplus force to improve the system loading accuracy is a key technical problem of ELLS.

\subsection{Control methods}

\subsubsection{Position feedforward compensation}

In order to suppress the surplus force caused by the motion of the actuator, Structure invariability principle[9] as feedforward compensation to the disturbance is adopted to diminish the strong position disturbance of actuator, The linear grid ruler collects the displacement of the actuator in real-time, the feedforward compensator is designed. In theory, if the feedforward block $G_{2}$ satisfies the equation $G_{2}=G_{1} \times G_{q}$, The surplus force will be eliminated, The principle of position feedforward compensation is shown in Fig. 8.

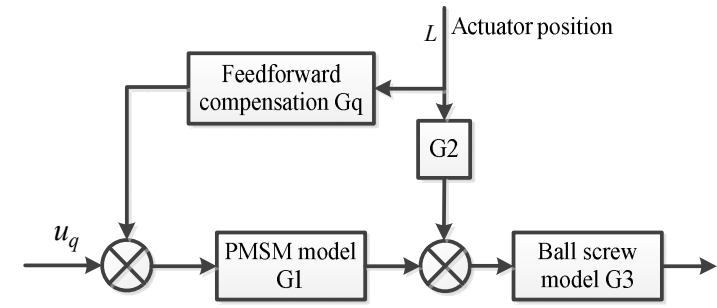

Fig. 8. Principle of position feedforward compensation.

\subsubsection{Input feedforward compensation}

In order to realize the adaptive adjustment of the force command signal according to the error signal, The force command feedforward compensation is introduced to improve the loading accuracy of the system.

\subsubsection{Force closed-loop PID controller}

In order to eliminate steady-state error of ELLS and further improve the loading accuracy of ELLS. The force sensor detects the linear force of the ELLS in real time, Then introduce PID to form force closed-loop control. PID can automatically reduce the error and improve the accuracy of the system.

\subsection{Control block diagram of ELLS}

Three control methods mentioned in section 4.2 are introduced into the control program of PMSM, and the control block diagram of ELLS is shown in Fig. 9.

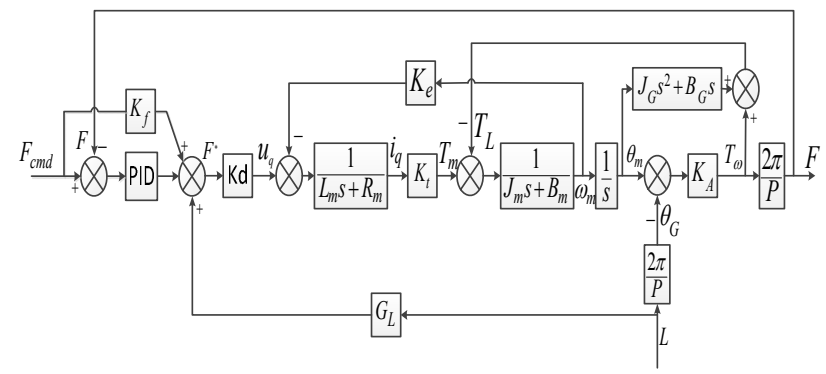

Fig. 9. Control block diagram of ELLS.

\section{Experimental results}

\subsection{Experimental setup configuration}

In order to verify the rationality and effectiveness of the design, The ELLS physical model was built and debugged. The final ELLS platform is shown in Fig. 10.

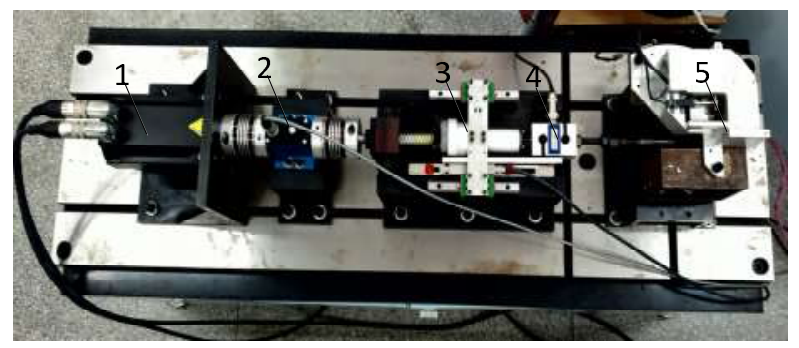

1-PMSM; 2-Torque speed sensor; 3-Ball screw; 4-Force sensor; 5-Actuator

Fig. 10. Photo of ELLS.

\subsection{Signal tracking experiment}

To test whether the dynamic loading accuracy of ELLS satisfies the "Double Ten Index" [10], let the ELLS tracks sinusoidal signal $1000 \mathrm{~N}-2 \mathrm{~Hz}$ while the actuator is operating with sinusoidal signal $1 \mathrm{~mm}-2 \mathrm{~Hz}$. Then let ELLS tracks $1000 \mathrm{~N}-5 \mathrm{~Hz}$ while the actuator tracks $1 \mathrm{~mm}-$ $5 \mathrm{~Hz}$. The loading force tracking curves are shown in Fig. 11 and Fig. 12.

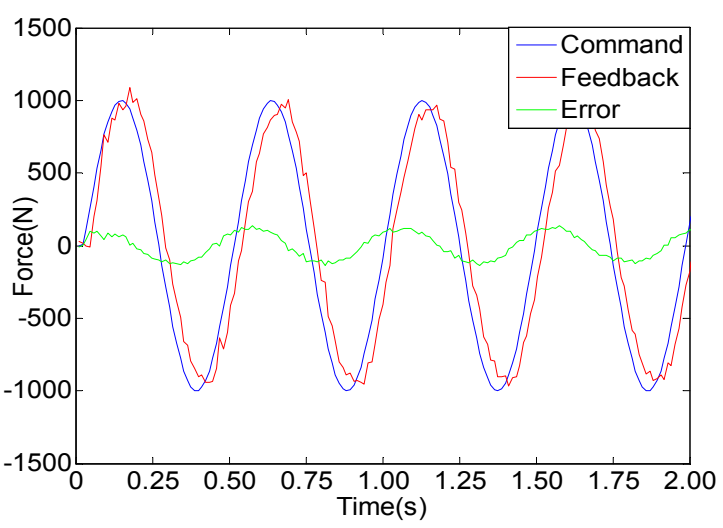

Fig. 11. Force tracks $1000 \mathrm{~N}-2 \mathrm{~Hz}$ with actuator tracking $1 \mathrm{~mm}-$ $2 \mathrm{~Hz}$. 


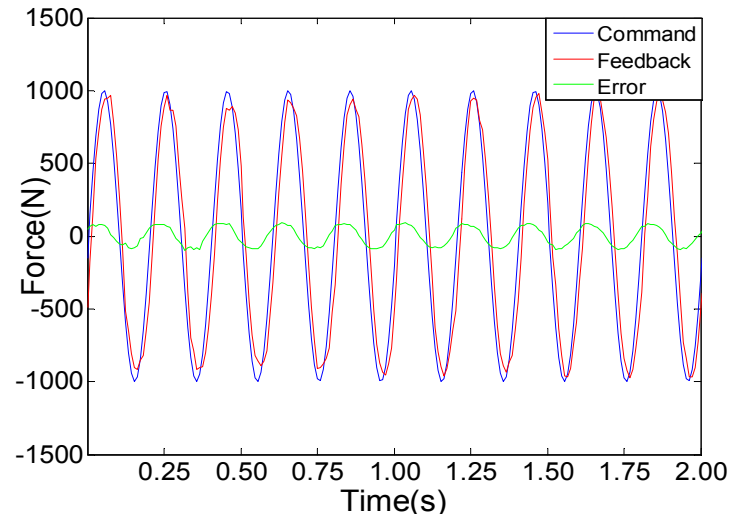

Fig. 12. Force tracks $1000 \mathrm{~N}-5 \mathrm{~Hz}$ with actuator tracking $1 \mathrm{~mm}-$ $5 \mathrm{~Hz}$.

In fig. 11, when ELLS tracks $1000 \mathrm{~N}-2 \mathrm{~Hz}$ with actuator tracking $1 \mathrm{~mm}-2 \mathrm{~Hz}$, the positive peak value of the feedback curve is $1075.45 \mathrm{~N}$, and the reverse peak value is $-1063.38 \mathrm{~N}$, the loading accuracy is up to $93.5 \%$; Furthermore, in fig. 12, when ELLS tracks $1000 \mathrm{~N}-5 \mathrm{~Hz}$ with actuator tracking $1 \mathrm{~mm}-5 \mathrm{~Hz}$, the loading accuracy is up to $90.3 \%$.

\section{Conclusion}

In order to test a certain type of electric linear actuator, a design scheme of electric linear load simulator (ELLS) based on LabVIEW+PXI is proposed. in structural design, the scheme of PMSM+ball screw is adopted to load the linear actuator; in hardware design, the system is built using the upper-lower computer system architecture based on PXI bus; The program is written in LabVIEW; furthermore, control strategies such as position feedforward compensation, input feedforward compensation and PID controller are adopted in PMSM control program. Finally, the experimental results showed that the loading accuracy of ELLS reaches $93.5 \%$ when tracking $1000 \mathrm{~N}-2 \mathrm{~Hz}$, and $90.3 \%$ when tracking $1000 \mathrm{~N}-5 \mathrm{~Hz}$. The force output error of ELLS is less than $10 \%$, which satisfies the "Double Ten Index".

\section{References}

1. C. Wang, Z. Jiao, et al. An experimental study of the dual-loop control of electro-hydraulic load simulator (EHLS), Chinese Journal of Aeronautics, 26(6): 1586-1595 (2013).

2. D. Wang, Y. ZHANG, et al. Displacement detection system of industrial controller based on LabVIEW, Manufacturing Automation, 40(5): 11-14 (2018).

3. J. Li, Z. Peng, M. Luo. Testing Methods for Performance of Steering Gear System Based on LabVIEW, 2016 IEEE Chinese Guidance, Navigation and Control Conference, 802-806 (2016).

4. J. Wang. Design of Simulation Loading Test System Based on Virtual Instrument. Nanjing: Nanjing University of Science and Technology, 6 (2012).

5. Z. Li, Y. Shang, et al. Surplus Torque Elimination Control of Electro-Hydraulic Load Simulator Based on Actuator Velocity Input Feedforward Compensating Method, Journal of Dynamic Systems, Measurement, and Control, 140(10): 101001101001-8 (2018).

6. L. Wang, M. Wang, et al. A Loading Control Strategy for Electric Load Simulators Based on Proportional Resonant Control, IEEE Transactions on Industrial Electronics, 65(6): 4608-4618 (2018).

7. S. Abu. Design methodologies for robust nanopositioning, IEEE Transactions on Control Systems Technology, 13(6): 868 876 (2005).

8. C. Li, H. Jin, Z. Jiao. Mechanism and suppression of extraneous torcue of motor driver load simulator, JournaI of Beijing University of Aeronautics and Astronautics, 32(2), 204 208 (2006).

9. S. J. Elliott, T. J. Sutton. Performance of Feedforward and Feedback Systems for Active Control, IEEE Trans. on Speech and Audio Processing, 4(3): 214 223 (1996).

10. Z. Jiao, Q. Hua, X. Wang. Estimation for Performance of Load Simulator, Journal of Mechanical Engineering, 38(11): 26 30 (2002). 